\title{
HISTERECTOMÍA LAPAROSCÓPICA TOTAL, CURVA DE APRENDIZAJE: EXPERIENCIA DE CLÍNICA DEL PRADO, MEDELLÍN, COLOMBIA
}

\author{
Eduardo Serna A. ${ }^{1}$, Giovanni Riaño C. ${ }^{2}$, Luis Almanza P. ${ }^{1}$, José De Los Ríos P. ${ }^{1}$, Juan \\ Castañeda R. ${ }^{1}$, Gustavo Calle G. ${ }^{1}$, Ricardo Vásquez R. ${ }^{1}$, Adriana Arango M. ${ }^{1}$, Ana \\ Mejía $B .^{a}$ \\ 1 Unidad de Endoscopia Ginecológica, Clínica del Prado; ${ }^{2}$ Programa de Cirugía Endoscópica Ginecológica; Universidad \\ CES, Medellín, Colombia.
}

\footnotetext{
a Instrumentadora Quirúrgica, Unidad de Endoscopia Ginecológica, Clínica del Prado, Universidad de Antioquia, Medellín, Colombia.
}

\section{RESUMEN}

Objetivos: Comparar la curva de aprendizaje en histerectomía laparoscópica total (HLT) de 4 ginecólogos en la Clínica del Prado, Medellín, Colombia. Determinar el número de cirugías necesarias para alcanzar un tiempo quirúrgico promedio de 90 minutos, disminuir la laparoconversiones a un $3 \%$ o menos y disminuir las complicaciones totales a menos del 10\%. Método: Se realizó un estudio de cohorte prospectivo que incluyó 626 pacientes operadas por 4 ginecólogos laparoscopistas con técnica y equipamiento quirúrgico similar. Se formaron 4 grupos de pacientes de acuerdo al orden en la casuística de cada uno de ellos. Resultados: El tiempo quirúrgico promedio de 93 minutos se logra con las primeras $50 \mathrm{HLT}$. El realizar entre 50-100 HLT baja las tasas de laparoconversión a 1,9\%. La tasa de complicaciones en la sumatoria de la primera y segunda cohorte es de 19,4\% y la tasa de complicaciones totales en la tercera cohorte es de 8,7\% (RR: 0,45; IC95\%: 0,26-0,78) la cual permanece en el límite deseado (10\%) en la cuarta cohorte. Conclusiones: La curva de aprendizaje en HLT para lograr un promedio de tiempo de cirugía de 90 minutos se logra con las primeras 50 histerectomías. A pesar de las limitaciones metodológicas, el presente estudia revela que para lograr la destreza máxima toma entre 50-100 histerectomías para lograr laparoconversiones menores del $3 \%$ y entre $100-150$ histerectomías para lograr complicaciones totales inferiores a $10 \%$.

\section{PALABRAS CLAVE: Histerectomía laparoscópica total, curva de aprendizaje, complicaciones quirúrgicas}

\section{SUMMARY}

Objective: To compare the learning curve in total laparoscopic hysterectomy (HLT) from four gynecologists in the Clinica del Prado, Medellín, Colombia. To determine the number of surgeries required to achieve an average surgical time of 90 minutes, to reduce laparoconversions to $3 \%$ or less and to reduce total complications to less than $10 \%$. Method: We performed a prospective cohort study including 626 patients operated by four gynecologists laparoscopist with similar technique and surgical equipment. We made four groups of patients according to the order in the cases of each one. Results: The mean operative time of 93 minutes is achieved with the first $50 \mathrm{HLT}$. Doing 50-100 HLT, laparoconversions rates low to $1.9 \%$. The complication rate in the sum of first and second cohort is $19.4 \%$ and the total complication rate in third cohort is $8.7 \%$ (RR: 
0.45; $95 \% \mathrm{Cl} 0.26-0.78)$ which remains in the desired limit (10\%) in fourth cohort. Conclusions: HLT learning curve to achieve an average surgical time of 90 minutes is achieved with the first 50 hysterectomies. Despite the methodological limitations this study, shows that to achieve maximum expertise, it takes between 50100 hysterectomies to achieve laparoconversions less than 3\%, and between 100-150 hysterectomies to achieve total complications lower than $10 \%$.

\section{KEY WORDS: Laparoscopic hysterectomy, learning curve, surgical complications}

\section{INTRODUCCIÓN}

Se considera una histerectomía laparoscópica total (HLT) aquella en la que todo el procedimiento, incluyendo el cierre de la cúpula vaginal, se hace por vía endoscópica.

Es necesario evaluar nuestra experiencia, más aún cuando se tienen estudiantes de posgrado en formación. El concepto de "curva de aprendizaje" fue introducido originalmente en la fabricación de aviones por Wright, quien describió una teoría básica para evaluar la producción repetitiva en ensamblajes de aeronaves $(1936)(1,2)$.

Una forma reproducible de definir una "curva de aprendizaje" es el tiempo y el número de procedimientos que un cirujano corriente, necesita para realizar un procedimiento en forma independiente, con un resultado razonable en tiempo y en complicaciones. Para alcanzar la destreza de hacer histerectomías laparoscópicas, el estándar se encuentra en 30-50 procedimientos, datos extraídos de múltiples estudios descriptivos y escasos estudios analíticos (3-12).

El objetivo de este estudio es determinar el número de HLT necesarias para alcanzar un tiempo quirúrgico promedio de 90 minutos, disminuir las laparoconversiones a un $3 \%$ o menos y disminuir las complicaciones totales por debajo del $10 \%$, datos estos obtenidos de promedios de diferentes estudios (13-15).

\section{MATERIAL Y MÉTODO}

Se analizaron las primeras $626 \mathrm{HLT}$, realizadas entre diciembre de 2002 y abril de 2008 que cumplían los siguientes criterios de inclusión: pacientes llevadas a HLT por patología ginecológica benigna, intervenidas por ginecólogos laparoscopistas parecidos en cuanto a su formación, uso de técnicas quirúrgicas, equipos y ayudantes con características similares, facilitando el control de sesgos. Se evaluó el tiempo quirúrgico promedio, los porcentajes de laparoconversión, complicaciones mayores y menores (sin tener en cuenta las complicaciones anestésicas) mediante una base de datos construi- da en EPlinfo basados en el análisis de las historias clínicas y el llamado periódico de las pacientes.

La técnica utilizada ha sido descrita en otra publicación y, brevemente, fue la siguiente (15): Los procedimientos se realizaron bajo anestesia general endotraqueal en posición de dorso litotomía. Todos los pacientes recibieron profilaxis con cefazolina $2 \mathrm{~g}$ por vía intravenosa, aproximadamente 30 minutos antes de la cirugía. Se realizó un tiempo vaginal en el cual se colocó el movilizador uterino y colpotomizador tipo VCare ${ }^{\circledR}$. Una aguja de Veress se insertó a través del ombligo, insuflando el abdomen con $\mathrm{CO} 2$, trabajando con presión intraabdominal de $15 \mathrm{~mm} \mathrm{Hg}$. Se insertó un trocar de $10 \mathrm{~mm}$ umbilical por el cual se introdujo el laparoscopio, y además tres trocares suprapúbi$\cos$ (2 pararectales izquierdos y uno derecho), por donde se introdujeron los instrumentos auxiliares. La pelvis y el acceso a los pedículos uterinos al igual que la visualización del colpotomizador fueron evaluados. Se realizó coagulación con bipolar 25 watts y corte de ligamentos redondos, trompas de Falopio y ligamentos uteroovaricos, logrando así abrir las dos hojas de ambos ligamentos anchos, continuándo la disección hasta lograr abrir el peritoneo anterior y posterior del útero. El espacio prevesical se abrió con la disección de la vejiga: se realizó tracción peritoneal hacia arriba lo cual creó una hoz peritoneal, que permitió coagular y seccionar el tejido laxo y desarrollar dicho espacio. Una vez que el plano fue abordado, los pilares de la vejiga se coagularon y se cortaron; la disección de la vejiga se continuó lo suficiente para exponer de manera clara la fascia endopélvica y el colpotomizador. En la parte posterior, el peritoneo y los ligamentos úterosacros se coagularon y disecaron bilateralmente. En ese momento se aplicó coagulación con bipolar 25 watts y corte bilateral de la rama ascendente de la arteria y vena uterina, a nivel de los bordes del fondo de saco vaginal expuestos por el colpotomizador y previa identificación del recorrido de ambos uréteres. La colpotomía se realizó con corte de 360 grados con corriente monopolar. El útero se extrajo parcialmente a través de la vagina, dejándose 
como neumotaponador para evitar la pérdida del CO2. Después de una hemostasia meticulosa, la vagina se cerró por vía laparoscópica en dos planos (mucosa en primer plano y afrontamiento de ligamentos uterosacros a fascia endopélvica y cierre del anillo pericervical en segundo plano), técnica continua con poliglactina 910 calibre 0 y aguja curva de $30 \mathrm{~mm}$, nudos intracorpóreos. Lavado de la cavidad pélvica con cristaloides y revisión de la integridad y movilidad de los uréteres y de la sutura vaginal. Todas las pacientes fueron evaluadas en el posoperatorio inmediato (entre día 7-15) para ver sus resultados en las variables descritas. Posteriormente fueron llamadas telefónicamente al mes para evaluar complicaciones tardías.

Se formaron 4 grupos: Destreza 1 (las primeras 50 de cada uno de los 4 ginecólogos, $n=200$ ), Destreza 2 (las segundas 50 de tres de ellos, uno de los ginecólogos aportó 6 histerectomías en esta cohorte, $n=156$ ), Destreza 3 (las terceras 50 de tres ginecólogos, $n=150$ ) y Destreza 4 (las cuartas 50 de uno de ellos, otro de los ginecólogos aportó 48 casos y el tercero aportó 22 histerectomías en esta cohorte, $n=120$ ).

El grupo discutió ampliamente en su interior, con epidemiólogos y estadísticos externos si este diseño de estudio era realmente de cohortes o de serie de casos. Se concluyó que si eran cohortes pues eran grupos similares que solo los diferenciaba el factor de riesgo destreza (número de HLT realizadas).

Se evaluó la destreza como la habilidad necesaria para lograr un tiempo quirúrgico promedio de 90 minutos (desde que se aplica el movilizador uterino y colpotomizador hasta que se cierra la piel). Evaluamos además la habilidad cuando se disminuye a un $3 \%$ la necesidad de laparoconversión, variable esta que evalúa la capacidad del clínico de escoger la paciente adecuada y de tener la destreza necesaria para lograr completar la cirugía por vía laparoscópica. Las complicaciones fueron evaluadas según Garry y cols (eVALuate study) (13), para lograr un estándar acorde a la literatura internacional. Complicaciones mayores: herida del tracto urinario, hemorragias que requirieron transfusión, heridas vasculares e intestinales, hematomas que requirieron drenaje, dehiscencia de sutura; complicaciones menores: desgarros vaginales, infecciones de cúpula, infecciones de pared abdominal, infecciones urinarias, sangrado tardío de cúpula, hematomas sin drenaje. A diferencia del eVALuate study no consideramos la laparoconversión como una complicación mayor, pues creemos que esto genera un sesgo en la evaluación de complicaciones mayores y totales. El laparoconvertir no es una complicación sino un cambio en la vía de abordaje por dificultades técnicas, y no tiene comparación con la abdominal pues en ella el resultado siempre será cero, razón por la cual la consideramos como una variable independiente, importante a tener en cuenta como un desenlace aparte.

No se consideraron las complicaciones anestésicas, ya que estas no evalúan la curva de aprendizaje técnico del cirujano y generarían sesgos al comparar las complicaciones totales, pues pueden depender de otras variables diferentes a la destreza lograda por este.

Se compararon las 4 cohortes para identificar un punto de corte en el cual se lograra un tiempo quirúrgico de 90 minutos o menor, un porcentaje de laparoconversiones menor al $3 \%$ y las complicaciones totales menores al 10\% (13-15).

Las variables demográficas fueron comparadas empleando el estadístico $t$, al evaluar la diferencia de medias en las variables continuas y se utilizó el estadístico $z$ al comparar las variables cualitativas. Se utilizó el paquete estadístico Epidat Versión 3.1. Se compararon las variables dicotómicas de los desenlaces a medir y se realizo un análisis con riesgos relativos con sus respectivos intervalos de confianza y su valor $p$. Un valor de $p<0,05$ se consideró estadísticamente significativo.

Este estudio se clasifica como de riesgos mínimos, es decir, no afecta la intimidad de las personas y está cumpliendo con lo dispuesto en la Resolución 0843 de 1993 del Ministerio de Salud de Colombia. No se incurrió en violaciones ya que la información fue tomada directamente de la historia clínica, se tuvo en reserva la identidad de los pacientes, de acuerdo a la declaración de Helsinki y la ley 23 de 1981 (ley de ética médica colombiana).

\section{RESULTADOS}

La edad, la paridad, el antecedente de cesárea, el riesgo quirúrgico evaluado por la clasificación ASA, el peso uterino y la indicación del procedimiento son similares en las cuatro cohortes, haciéndolas comparables (Tabla I). El peso corporal entre los grupos es similar, solo existiendo diferencia significativa entre la primera y la cuarta cohorte con diferencia de medias de 2,7 kg (IC95\% 0,393 - 5,067 $\mathrm{kg}$ ), pero sin significancia clínica. La diferencia en cuanto indicación quirúrgica por lesiones preinvasivas cervicales tiene significancia estadística, pero no la tiene desde el punto de vista clínico, pues esto 


\section{Tabla I}

\section{COMPARACIÓN DE LAS CARACTERÍSTICAS DE LAS PACIENTES EN CADA UNO DE LOS GRUPOS}

\begin{tabular}{|c|c|c|c|c|c|}
\hline & $\begin{array}{c}\text { Primeras } 200 \\
\text { histerectomías }\end{array}$ & $\begin{array}{l}\text { Segundas } 156 \\
\text { histerectomías }\end{array}$ & $\begin{array}{c}\text { Terceras } 150 \\
\text { histerectomías }\end{array}$ & $\begin{array}{c}\text { Cuartas } 120 \\
\text { histerectomías }\end{array}$ & Valor $p$ \\
\hline Edad (años) & $(\mathrm{n}=200)$ & $(n=156)$ & $(n=150)$ & $(n=120)$ & \\
\hline Promedio & $42,7 \pm 6,4$ & $42,9 \pm 6,8$ & $42,3 \pm 6,0$ & $42,6 \pm 6,6$ & $N S^{*}$ \\
\hline Peso (kilos) & $(\mathrm{n}=198)$ & $(n=154)$ & $(n=149)$ & $(n=120)$ & \\
\hline Promedio & $61,8 \pm 9,7$ & $63,3 \pm 10,6$ & $62,7 \pm 9,5$ & $64,6 \pm 11,1$ & $0,02^{\star a}$ \\
\hline Paridad & $(\mathrm{n}=200)$ & $(\mathrm{n}=156)$ & $(\mathrm{n}=150)$ & $(n=120)$ & \\
\hline Promedio & $2,2 \pm 1,8$ & $2,2 \pm 1,5$ & $2,4 \pm 1,3$ & $2,5 \pm 1,6$ & $\mathrm{NS}^{*}$ \\
\hline $\begin{array}{l}\text { Cesáreas } \\
(\%)\end{array}$ & $\begin{array}{c}(n=200) \\
16,5\end{array}$ & $\begin{array}{c}(\mathrm{n}=156) \\
16,0\end{array}$ & $\begin{array}{c}(n=150) \\
15,3\end{array}$ & $\begin{array}{c}(n=119) \\
13,5\end{array}$ & $N S^{\&}$ \\
\hline ASA & $(n=199)$ & $(n=155)$ & $(n=150)$ & $(n=120)$ & \\
\hline ASA 1 (\%) & 84,0 & 83,2 & 86,6 & 78,3 & $N S^{\&}$ \\
\hline ASA 2 (\%) & 15,5 & 16,1 & 13,3 & 20,8 & \\
\hline Patología (\%) & $(n=195)$ & $(n=150)$ & $(n=149)$ & $(n=116)$ & \\
\hline Miomatosis & 58,0 & 56,0 & 59,7 & 57,8 & $N S^{\&}$ \\
\hline Adenomiosis & 13,3 & 12,7 & 10,0 & 13,8 & $N S^{\&}$ \\
\hline $\mathrm{NIC}$ & 7,7 & 4,6 & 4,0 & 0,8 & $0,01^{\& b}$ \\
\hline Otros & 21,0 & 26,6 & 26,8 & 27,6 & $N S^{\&}$ \\
\hline Peso útero (g) & $(n=85)$ & $(n=121)$ & $(n=132)$ & $(n=103)$ & \\
\hline Promedio & $182,0 \pm 97,1$ & $182,0 \pm 90,4$ & $187,0 \pm 112,1$ & $179,0 \pm 73,0$ & $\mathrm{NS}^{\star}$ \\
\hline
\end{tabular}

ASA: Evaluación prequirúrgica del riesgo anestésico, según la American Society of Anesthesiologists. NIC: Neoplasia Intraepitelial Cervical. ${ }^{a}$ Diferencia estadísticamente significativa al comparar cohorte 1 vs $4 .{ }^{\text {b }}$ Diferencias estadísticamente significativas al comparar cohorte 1 vs $4 .{ }^{\&}$ Diferencia de medias utilizando estadístico t. * Diferencia de proporciones utilizando estadístico $z$.

no influye en los desenlaces.

En la Tabla II, se observa que los ginecólogos lograron el tiempo de 93 minutos en las primeras 50 histerectomías. Se muestra además que con mayor experiencia se logra bajar significativamente el promedio del tiempo quirúrgico en 12,6 minutos (IC95\%: 6,515-18,745 minutos), cuando se compara el grupo de las 50 primeras HLT con el grupo de 151 a 200 HLT. Sin embargo, la diferencia empieza a ser sustancial cuando se compara la primera contra la segunda cohorte (10,1 minutos; IC95\%: 4,88-15,38 minutos).

Para ver si es posible adquirir el tiempo estándar de cirujano en una cantidad de cirugías menores, se realizó un recálculo con las primeras 25 HLT de cada uno de los 4 ginecólogos laparoscopistas (100 histerectomías), encontrando un promedio de 97 minutos \pm 31 minutos.

En la pérdida sanguínea intraquirúrgica, tam- bién se observó una disminución de $19 \mathrm{ml}$ entre el segundo y el tercer grupo (IC95\%: 1,6-36,6 ml), diferencia que se mantiene al compararse con la cuarta cohorte (18,5 ml; IC95\%: 2,1-34,8 ml), lo que muestra la adquisición de mayor destreza, pero que no amerita estudios estadísticos adicionales, pues no tiene una relevancia clínica. Esta medida se realizó de manera subjetiva (calculada por el cirujano) lo cual puede generar sesgos.

En cuanto a los índices de laparoconversión se encontró que entre la primera y segunda cohorte no hay diferencias estadísticamente significativas (RR: 0,55; IC95\%: 0,14-2,09) (Tabla III). Al evaluar la sumatoria de la primera y segunda cohorte vs la tercera encontramos un RR: 0,95 (IC95\%: 0,30-2,98). Tampoco hay diferencia al comparar la sumatoria de la primera y segunda cohorte contra la tercera y la cuarta. La tasa de laparoconversiones menores al $3 \%$ se logra después de las primeras 50 histerec- 


\section{Tabla II}

TIEMPO DEL CIRUJANO Y SANGRADO QUIRÚRGICO EN LAS DIFERENTES COHORTES

\begin{tabular}{lccccc}
\hline & $\begin{array}{c}\text { Primeras 200 } \\
\text { histerectomías }\end{array}$ & $\begin{array}{c}\text { Segundas 156 } \\
\text { histerectomías }\end{array}$ & $\begin{array}{c}\text { Terceras 150 } \\
\text { histerectomías }\end{array}$ & $\begin{array}{c}\text { Cuartas 120 } \\
\text { histerectomías }\end{array}$ & Valor $p$ \\
\hline Tiempo cirujano (min) & $(\mathrm{n}=200)$ & $(\mathrm{n}=155)$ & $(\mathrm{n}=150)$ & $(\mathrm{n}=119)$ & $<0,05^{\star a}$ \\
Promedio & $93,23 \pm 29,8$ & $83,1 \pm 20,4$ & $83,4 \pm 34,9$ & $80,6 \pm 24,9$ & $(\mathrm{n}=120)$ \\
Pérdida sanguínea $(\mathrm{ml})$ & $(\mathrm{n}=199)$ & $(\mathrm{n}=156)$ & $(\mathrm{n}=150)$ & $52,6 \pm 57,9$ & $<0,05^{\star b}$ \\
Promedio & $75,4 \pm 60,8$ & $71,1 \pm 80,0$ & $52,0 \pm 75,3$ & 5 \\
\hline
\end{tabular}

* Diferencias de medias utilizando estadístico t. a Valor $\mathrm{p}<0,05$ al comparar cohorte 1 vs 2 , al comparar cohorte 1 vs 3 y al comparar cohorte 1 vs 4 . No hay diferencias significativas en las demás comparaciones. ${ }^{b}$ Valor $p<0,05$ al comparar cohorte 1 vs 3 , al comparar cohorte 2 vs 3 , al comparar cohorte 1 vs 4 y al comparar cohorte 2 vs 4 . Sin diferencias significativas en las demás comparaciones.

Tabla III

\section{LAPAROCONVERSIONES Y COMPLICACIONES MAYORES Y MENORES EN LAS DIFERENTES COHORTES}

\begin{tabular}{|c|c|c|c|c|c|c|c|c|}
\hline & $\begin{array}{l}\text { Primeras } 200 \\
\text { histerectomías }\end{array}$ & $\%$ & $\begin{array}{l}\text { Segundas } 156 \\
\text { histerectomías }\end{array}$ & $\%$ & $\begin{array}{l}\text { Terceras } 150 \\
\text { histerectomías }\end{array}$ & $\%$ & $\begin{array}{l}\text { Cuartas } 120 \\
\text { histerectomías }\end{array}$ & $\%$ \\
\hline Laparoconversión & 7 & 3,5 & 3 & 1,9 & 4 & 2,7 & 0 & 0 \\
\hline \multicolumn{9}{|l|}{ Complicaciones mayores } \\
\hline Herida vejiga & 2 & 1,0 & 0 & 0 & 1 & 0,7 & 0 & 0 \\
\hline Herida intestinal & 0 & 0 & 1 & 0,6 & 0 & 0 & 0 & 0 \\
\hline Transfusión & 4 & 2,0 & 4 & 2,6 & 2 & 1,3 & 1 & 0,8 \\
\hline Total mayores & 6 & 3,0 & 5 & 3,2 & 3 & 2,0 & 1 & 0,8 \\
\hline \multicolumn{9}{|l|}{ Complicaciones menores } \\
\hline Infección cúpula & 12 & 6 & 10 & 6,4 & 5 & 3,3 & 6 & 5,0 \\
\hline Infección pared abdominal & 2 & 1,0 & 2 & 1,3 & 1 & 0,7 & 0 & 0 \\
\hline Infección urinaria & 5 & 2,5 & 3 & 1,9 & 2 & 1,3 & 2 & 1,7 \\
\hline Hemorragia tardía & 4 & 2,0 & 6 & 3,8 & 0 & 0 & 1 & 0,8 \\
\hline Fístula líquido & 1 & 0,5 & 0 & 0 & 0 & 0 & 0 & 0 \\
\hline Desgarro vaginal & 0 & 0 & 1 & 0,6 & 0 & 0 & 1 & 0,8 \\
\hline Hematomas & 6 & 3,0 & 6 & 3,8 & 2 & 1,3 & 1 & 0,8 \\
\hline Total menores & 30 & 15,0 & 28 & 17,9 & 10 & 6,7 & 11 & 9,2 \\
\hline Totales & 36 & 18,0 & 33 & 21,2 & 13 & 8,7 & 12 & 10,0 \\
\hline
\end{tabular}

tomías y se mantiene en las otras cohortes.

La tasa de complicaciones mayores es del 3,0\% (sin tener en cuenta las complicaciones anestésicas) con las primeras 50 cirugías, muy similar a las reportadas en la literatura mundial (15). En las cohortes siguientes se va haciendo más baja, diferencias estas que no son estadísticamente significativas. No hay diferencia entre la primera y segunda cohorte en cuanto a complicaciones menores (RR: 1,20; IC95\%: 0,75-1,92). Se decidió comparar la suma de las complicaciones menores de la primera y segunda cohorte contra la tercera cohorte, encon- trando un RR: 0,41 (IC95\%: 0,22-0,78) con un NNT (número necesario a tratar para evitar una complicación menor) de 10. Las complicaciones menores de la primera y segunda cohorte, comparadas contra la suma de la tercera y cuarta cohorte, reporta una diferencia significativa (RR: 0,48; IC95\%: 0,300,77) con un NNT 12.

No hay diferencia en cuanto a las complicaciones totales entre la primera y segunda cohorte. Si comparamos las complicaciones totales de la suma de la primera y segunda cohorte comparadas contra la tercera cohorte encontramos un RR: 0.45 (IC 
95\%0.26-0.78) con un NNT 9. Las complicaciones totales de la primera y segunda cohorte comparadas contra la suma de la tercera y cuarta cohorte revela un RR: 0,48 (0,31-0,73) con un NNT de 10.

\section{DISCUSIÓN}

Para implementar la histerectomía laparoscópica vs la abdominal se deben sopesar los beneficios sobre los riesgos: menos infecciones totales, menos estancia hospitalaria, menor tiempo en asumir las actividades diarias vs más complicaciones de vías urinarias, más tiempo quirúrgico (14). Las desventajas se pueden disminuir logrando una destreza mayor.

Como la balanza entre unos y otros es tan frágil hay que garantizar que si se va a introducir dicha tecnología se tenga la mayor destreza posible. Surge entonces el término de curva de aprendizaje, el cual se basa en dos ítems: tiempo quirúrgico y disminución de las complicaciones, que reflejan las habilidades técnicas (coordinación, destreza) y la capacidad de tomar decisiones en momentos críticos.

Según Hopper y cols (16), se debe trazar un estándar para decidir cuál es el momento óptimo de lograr la destreza en cualquier entrenamiento en educación médica (Figura 1). La parte difícil de decidir es si el estándar es el tiempo quirúrgico, o cuando se logran tasas de complicaciones razonables. Veamos que se ha dicho al respecto en otras áreas del aprendizaje quirúrgico (Tabla IV). Se observa que las tasas de complicaciones, el tiempo quirúrgico y las laparoconversiones han sido los desenlaces más importantes a evaluar en la construcción de la curva de aprendizaje.

Tal vez el mejor trabajo para responder la pregunta tiempo es el de Perino y cols (12). Corresponde a un estudio clínico controlado que muestra la comparación del tiempo quirúrgico en las curvas de aprendizaje entre las primeras histerectomías abdominales y laparoscópicas totales. Al realizar cortes a las 15 y 51 cirugías, encuentra que la curva se estabiliza en la número 15 (87 minutos) en la histerectomía abdominal; no así en la laparoscópica la cual toma 129 minutos en las primeras 15, baja a 93,6 minutos entre la 16 y la 51 , siendo en este punto similar en tiempo con el grupo de histerectomía abdominal. El tamaño muestral no le permitió evaluar otros desenlaces importantes como son las complicaciones.

Brummer y cols (17), evaluaron este tema en Finlandia en un gran estudio retrospectivo, en el que consideraron 27.827 histerectomías laparoscópicas encontrando que la tasa de complicaciones mayores se reduce de manera significativa a me-

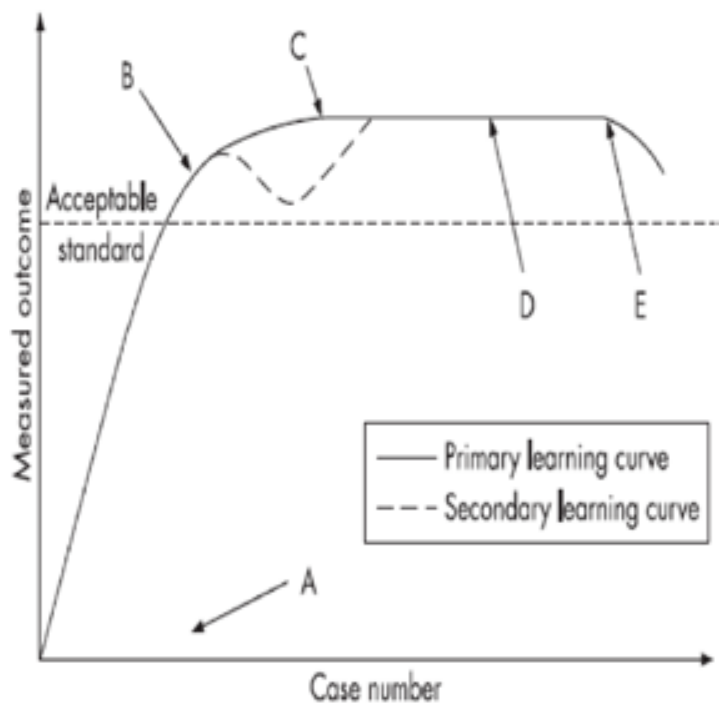

Figura 1. Curva de aprendizaje quirúrgico ideal. A: es la parte inicial del entrenamiento; $\mathrm{B}$ : cuando se es capaz de hacerlo con competencia e independiencia; C: experiencia adicional mejora levemente los resultados; D: se logra la parte plana de la curva; E: decadencia por la edad o por la falta de estar haciendo dichos procedimiento. La línea punteada representa un deterioro temporal del desempeño al asumir casos de mayor complejidad o exceso de confianza (Reproducida y adaptada de Learning Curves in Surgical Practice. Hopper y cols. Postgrad Med J 2007;83:777-9, con permiso de BMJ Publishing Group Ltd. Licencia número 2437220975692).

dida que se aumenta el número de procedimientos practicados, según una curva de aprendizaje nacional. Entre 1992 al 2005 la incidencia de todas las complicaciones mayores disminuyó un $74 \%$ (en promedio un $11 \%$ por año), las lesiones del tracto urinario un $83 \%$ (en promedio un $13 \%$ por año) y las lesiones ureterales en un $79 \%$ (en promedio un $11 \%$ por año), y desde el año 1999 la tasa de complicaciones fue razonablemente estable, habiéndose logrado una curva de aprendizaje nacional para ese año.

Mäkinen y cols (5), en 10.110 histerectomías encontraron que las complicaciones tales como lesión ureteral y lesión vesical son menores en histerectomía laparoscópica de manera significativa cuando el cirujano ha realizado más de 30 procedimientos.

Al comparar nuestros resultados con los de Wattiez y cols (15), en su serie de 1.467 casos, se encuentra un tiempo quirúrgico promedio muy similar al reportado en su artículo en las segundas 952 pacientes operadas entre 1996-1999 con el ad- 
Tabla IV

MEDIDAS DE DESENLACES RELACIONADOS A LA DURACIÓN DE TIEMPO NECESARIO PARA LOGRAR COMPETENCIA

\begin{tabular}{|c|c|c|c|}
\hline Procedimiento quirúrgico & Desenlace & $\begin{array}{l}\text { Tiempo o número de } \\
\text { casos hasta la meseta }\end{array}$ & Referencia \\
\hline Apendicectomía laparoscópica & $\begin{array}{l}\text { Tasa de complicaciones, } \\
\text { conversión a procedi- } \\
\text { mientos abiertos, tasa de } \\
\text { reintervención. }\end{array}$ & 20 casos & Meinke y Kossuth (18) \\
\hline Cirugía colorrectal laparoscópica & $\begin{array}{l}\text { Conversión a procedi- } \\
\text { miento abierto, tasa de } \\
\text { complicación, tiempo } \\
\text { quirúrgico. }\end{array}$ & $55-80$ casos & Tekisy cols $(19,20)$ \\
\hline Colecistectomía laparoscópica & $\begin{array}{l}\text { Tasa de lesión de con- } \\
\text { ducto biliar. }\end{array}$ & 3 años & Richarson y cols (21) \\
\hline Gastrectomía D2 & $\begin{array}{l}\text { Morbilidad, mortalidad, } \\
\text { Extracción de GL. }\end{array}$ & $\begin{array}{l}18-24 \text { meses, } 15-25 \\
\text { casos }\end{array}$ & Parikh y cols (22) \\
\hline Esofagectomía & $\begin{array}{l}\text { Tiempo quirúrgico, pér- } \\
\text { dida sanguínea, estancia } \\
\text { en UCl, extracción de } \\
\text { ganglio linfático }\end{array}$ & $\begin{array}{l}\text { Mejoramiento continuo } \\
\text { por } 7 \text { años o } 150 \text { casos }\end{array}$ & Sutton y cols (23) \\
\hline Cirugía arteria coronaria & Mortalidad & 4 años & Bridgewater y cols (24) \\
\hline
\end{tabular}

Reproducida y adaptada de Learning Curves in Surgical Practice. Hopper y cols. Postgrad. Med. J. 2007;83:777-9, con permiso de BMJ Publishing Group Ltd. Licencia número 2437220975692.

venimiento de su movilizador uterino (90 minutos). Todas nuestras HLT fueron realizadas con movilizador uterino VCare ${ }^{\circledR}$.

La tasa de complicaciones mayores de nuestra serie fue inferior al $3 \%$ y la de complicaciones totales inferior al 10\% después de 100 histerectomías realizadas, similar a lo reportado en otras series $(25,26)$.

La tasa de laparoconversiones de nuestra serie fue inferior al $3 \%$ después de las primeras $50 \mathrm{HLT}$, acorde con los hallazgos en la literatura mundial (13).

Para nosotros es importante valorar las complicaciones menores, pues agregan valor a la tecnología laparoscópica según la conclusión de Cochrane, al garantizar menos infecciones de herida quirúrgica y morbilidad febril (14). El darle el nombre de complicaciones menores da una falsa impresión de no ser importantes, siendo otra la realidad: la histerectomía laparoscópica tiene esto como ventaja competitiva, variable entonces de importancia en la curva de aprendizaje.

\section{CONCLUSIONES}

El aporte este estudio a la literatura mundial, es establecer que para lograr controlar las complicaciones menores y totales se necesitan entre $100 \mathrm{y}$ 150 HLT. Según la curva de Hopper y cols (16), el cirujano promedio logra el punto de quiebre importante (punto B) a las 50 cirugías, pues controla el tiempo quirúrgico promedio y las complicaciones mayores. Logra un entrenamiento intermedio (punto C) entre 50-100 histerectomías, bajando las tasas de laparoconversión al 3\% y logrando el nivel de destreza máximo, al estabilizar las complicaciones totales y menores por debajo del $10 \%$ con 100 150 cirugías (punto D de la curva de Hopper).

Agradecimientos a la Universidad CES y al Dr José Bareño Silva magíster en epidemiología, especialista en estadística y docente de la Universidad, por su colaboración en el proceso estadístico de los datos; y a las directivas y personal médico y paramédico de la Clínica Del Prado, quienes con su trabajo y apoyo han permitido llevar a cabo esta investigación. 


\section{BIBLIOGRAFÍA}

1. Latiff A. La "Curva de Aprendizaje" ¿qué es y cómo se mide? Revista Urología Colombiana 2005;14:15-17.

2. Wright TP. Factors affecting the cost of airplanes. J Aeronautical Sciences 1936;3:122-8.

3. Fleisch M, Newton J, Steinmetz I, Whitehair J, Hallum A, Hatch K. Learning and teaching advanced laparoscopic procedures: Do alternating trainees impair a laparoscopic surgeon's learning curve? J Minim Invasive Gynecol 2007;14:293-9.

4. Ghomi A, Littman P, Prasad A, Einarsson JI. Assessing the learning curve for laparoscopic supracervical hysterectomy. JSLS 2007;11:190-4.

5. Mäkinen J, Johansson J, Tomás C, Tomás E, Heinonen $\mathrm{P}$, Laatikainen $\mathrm{T}$, et al. Morbidity of 10110 hysterectomies by type of approach. Hum Reprod 2001;16:1473-8.

6. Vaisbuch E, Goldchmit C, Ofer D, Agmon A, Hagay Z. Laparoscopic hysterectomy versus total abdominal hysterectomy: A comparative study. Eur J Obstet Gynecol Reprod Biol 2006;126:234-8.

7. Garrett A, Nascimento M, Nicklin J, Perrin L, Obermair A. Total laparoscopic hysterectomy: The Brisbane learning curve. Aust NZJ Obstet Gynaecol 2007;47:65-6.

8. Kolkman W, Engels LE, Smeets MJ, Jansen FW. Teach the teachers: an observational study on mentor traineeship in gynecological laparoscopic surgery. Gynecol Obstet Invest 2007;64:1-7.

9. Altgassen C, Michels W, Schneider A. Learning laparoscopic-assisted hysterectomy. Obstet Gynecol 2004;104:308-13.

10. Kreiker GL, Bertoldi A, Larcher JS, Orrico GR, Chapron C. Prospective evaluation of the learning curve of laparoscopic-assisted vaginal hysterectomy in a university hospital. J Am Assoc Gynecol Laparosc 2004;11:229-35.

11. Malzoni M, Perniola G, Perniola F, Imperato F. Optimizing the total laparoscopic hysterectomy procedure for benign uterine pathology. J Am Assoc Gynecol Laparosc 2004;11:211-8.

12. Perino A, Cucinella G, Venezia R, Castelli A, Cittadini E. Total laparoscopic hysterectomy versus total abdominal hysterectomy: an assessment of the learning curve in a prospective randomized study. Human Reprod 1999;14:2996-9.

13. Garry R, Fountain J, Mason S, Hawe J, Napp V, Abbott $\mathrm{J}$, et al. The eVALuate study: two parallel randomised trials, one comparing laparoscopic with abdo- minal hysterectomy, the other comparing laparoscopic with vaginal hysterectomy. BMJ 2004;328:129.

14. Johnson N, Barlow D, Lethaby A, Tavender E, Curr E, Garry R. Surgical approach to hysterectomy for benign gynaecological disease. Cochrane Database of Systematic Reviews 2006, Issue 2. Art. No.: CD003677. DOI: 10.1002/14651858.CD003677.pub3.

15. Wattiez A, Soriano D, Cohen S, Nervo P, Canis M, Botchorishvili $\mathrm{R}$, et al. The learning curve of total laparoscopic hysterectomy: comparative analysis of 1647 cases. J Am Assoc Gynecol Laparosc 2002;9:339-45.

16. Hopper AN, Jamison M, Lewis W. Learning curves in surgical practice. Postgrad Med 2007; 83:777-9.

17. Brummer T H, Seppälä $T$, Härkki $P$. National learning curve for laparoscopic hysterectomy and trends in hysterectomy in Finland 2000-2005. Hum Reprod 2008;23:840-5.

18. Meinke AK, Kossuth T. What is the learning curve for laparoscopic appendectomy? Surg Endosc 1994;8:371-5.

19. Tekkis PP, Fazio VW, Lavery IC, Remzi FH, Senagore AJ, Wu JS, et al. Evaluation of the learning curve in ileal pouch-anal anastomosis surgery. Ann Surg 2005;241:262-8.

20. Tekkis PP, Senagore AJ, Delaney CP, Fazio VW. Evaluation of the learning curve in laparoscopic colorectal surgery: comparison of right-sided and leftsided resections. Ann Surg 2005;242:83-91.

21. Richardson MC, Bell G, Fullarton GM. Incidence and nature of bile duct injuries following laparoscopic cholecystectomy: an audit of 5913 cases. West of Scotland Laparoscopic Cholecystectomy Audit Group. Br J Surg 1996;83:1356-60.

22. Parikh $D$, Johnson $M$, Chagla L, Lowe $D$, McCulloch $P$. D2 gastrectomy: lessons from a prospective audit of the learning curve. Br J Surg 1996;83:1595-9.

23. Sutton DN, Wayman J, Griffin SM. Learning curve for oesophageal cáncer surgery. Br J Surg 1998;85:1399402.

24. Bridgewater B, Grayson AD, Au J, Hassan R, Dihmis WC, Munsch C, Waterworth P. Improving mortality of coronary surgery over first four years of independent practice: retrospective examination of prospectively collected data from 15 surgeons. BMJ 2004;329:421.

25. Liu CY, Reich H. Complications of total laparoscopic hysterectomy in 518 cases. Gynaecol Endosc 1994;2:203-8.

26. Ou CS, Beadle E, Presthus J, Smith M. A multicentre review of 839 laparoscopic-assisted vaginal hysterectomies. J Am Assoc Gynecol Laparosc 1994;1:417-22. 